\title{
Towards the Generation of Bilingual Chinese-English Multi-word Expressions from Large Scale Parallel Cor- pora: An Experimental Approach
}

\author{
Benjamin K. Tsou ${ }^{1,3}$ Derek F. Wong ${ }^{2}$ Ka Po Chow ${ }^{3}$ \\ ${ }^{1}$ City University of Hong Kong \\ ${ }^{2}$ University of Macau \\ ${ }^{3}$ ChiLin (HK) Limited \\ btsou99@gmail.com
}

\begin{abstract}
There is increasing practical need for bilingual lexicon in wide ranging applications such as CAT systems and in cross-lingual information retrieval. The need for more sophisticated and more extensive resources involving bilingual multi-word expressions has increased even more for the bilingual processing of complex texts such as legal documents, technical names, contracts and patents. We report here a preliminary attempt at successful efforts to mine bilingual MWEs from 300+k Chinese-English patents.
\end{abstract}

Keywords: Parallel Corpus, Bilingual Alignment, Multiword Expressions.

\section{$1 \quad$ Introduction}

There is increasing practical need for bilingual glossaries in wide ranging applications such as CAT systems and in cross-lingual information retrieval. The need for more sophisticated and more extensive resources involving bilingual multi-word expressions has increased even more for the bilingual processing of complex texts such as legal documents, technical names, contracts and patents. Besides the availability of considerable computational power, the cultivation of the prequisite database has special requirements, such as, 1) Identification of thousands of relevant patent families across two languages; 2) Identification of parallel bilingual linguistic segments; 3) Evaluation of bilingual equivalence; 4) Pruning of poor results from the database, etc.

This task of data cultivation and curation is by no means a simple one, especially when comparability of Chinese and English patents was not easily determined. In addition to considerable noisy data, there are also considerable challenges in the bilingual sentence alignment during the cultivation of the training corpus on which the system is modelled. In a recent study based on a corpus of more than 300,000 "comparable" Chinese and English patents (Tsou 2017), it was found that the longest Chinese sentence had 1,417 words and the longest single document had 249,322 words in the wide-ranging collection of Chinese patents. The database had a total of more than 500 billion words and characters and 10.5 trillion characters, and on the average, there were 30,000 words and/or characters for each document. 
When the source language is Chinese, the patents have multiple additional challenges, such as: 1) Very long sentences consisting of continuous strings of characters; 2) Grammatical complexity: from frequent nominalization to telegraphic style; 3) Frequent subordination, implicit constructions, and dangling modifiers; 4) Problematical anaphora reference; 5) Active vs Passive forms; 6) incorrect characters; 7) Extensive neologisms; 8) Synonymy and polysemy; 9) wrong punctuation.

Words are conventionally segmented in the Western languages. However, an unusual challenge not faced by patents written in English, for example, but found in Chinese patent text is the opaque tokenization of the words represented by generally continuous strings of Chinese characters in texts. They are occasionally punctuated by a smaller set of punctuation marks than found in English, for example, but without the use of capitalization to delineate proper nouns or the use of italization to indicate special terms or emphasis. There is therefore a critical challenge with the proper tokenization and identification of name-entities and novel words or multi-word expressions are relative unique to Chinese text processing.

But the Chinese language has other uncommon problems with structural ambiguities beyond the classical "old man and woman" parallel, partly because of the combinatory possiblities of its mono-morpho-syllabic writing system.

There are still other challenges in Chinese patent processing, such as:

(a) Content formatting: Broken sentences; Wrong encoding; OCR errors

(b) Different data formatting: USPTO, EPO, WP, SIPO have own formats;

(c) Source of Data: Conversion from pdf into XML

(d) Text extraction from graphs and diagrams

Our efforts to obtain Chinese-English multiword expressions have several stages.

\section{Cultivation and curation of parallel corpus}

The successful development of corpora of bilingually aligned sentences has been made following extensive attempts involving the cultivation and curation of a sizable perquisite database of comparable Chinese and English patents. The English patent documents are harvested from the website of WIPO (World Intellectually Property Organization), while the Chinese patent documents are originated from SIPO, State Intellectual Property Office, the official patent office in China. The patent data is then divided into different sections, among which only title, abstract, claims and descriptions are our concern.

The correspondence between the English and Chinese patents are established through their cross-references. Since the comparable patents are not strictly parallel, some prior art of individual alignment methods might not be effective. For example, length-based alignment method might not be accurate since it does not consider content similarity. Moreover, new technical term poses a challenge to the bilingual dictionary-based alignment algorithm.

Following persistent efforts we have been able to harvest a database of $300+\mathrm{k}$ comparable Chinese-English patents. (Lu et al. 2009, 2011, 2015). 


\section{$3 \quad$ Filtering of bilingual sentence pairs}

We use a sequence of algorithms to filter good quality parallel sentence pairs. First we use a publicly available dictionary to perform preliminary alignment based on a dictionary-based similarity score of a sentence pair (Utiyama and Isahara, 2003).

In the resultant set of sentence pair candidates obtained from the above preliminary alignment, some pairs will be of very incomparable lengths. They are filtered based on a sentence length ratio which is found empirically. For example, if a sentence pair has around 50 words in the English sentence while there are more than 333 characters in the Chinese one, it is removed.

The parallel sentence candidates are further filtered by learning an IBM translation model Model-1 and the translation similarity score is computed by combining the translation probability value of both directions based on the trained model.

We make use of a number of existing alignment tools including Champollion (Ma 2006), Hunalign and Microsoft Sentence Aligner to arrive at a preliminary parallel by design if not on purpose corpus. Champollion applied dynamic programming based on a probabilistic score which allows us to obtain some correspondence other than 1to-1, like 1-to-2, 2-to-2, etc.

It should be noted that the resultant database of $10+$ million parallel sentence pairs are useful for training and fine-tuning MT system (Goto, 2013). However, they may not all be linguistically well-formed as they are the result of optimization of string matching (Koehn, 2010). For example, (1) “abdominal sac”-(膈 14下腹部 区域 内 的) 腹 囊; (2) “ability of the peptide” --多肽(单体解离的)能力; (3) “access time”--【访 问】时间.

\section{Extraction of bilingual multiword expressions}

As a further attempt to obtain linguistically well-formed entries, an alternative automatic approach to acquire the bilingual phrases from the above parallel corpus was made on the basis of Tian et al. $(2011,2014)$. We first derive the phrases from monolingual data, i.e. the source sentences of parallel data, by considering the possible $n$ gram of words. For those of high frequency words or phrases, we evaluate how likely a phrase can be constructed by two (words or) phrases $p_{i}$ and $p_{j}$, by considering the following significant score:

$$
\operatorname{Score}\left(P_{1}, P_{2}\right)=\frac{f\left(P_{1} \oplus P_{2}\right)-\mu_{0}\left(P_{1}-P_{2}\right)}{\sqrt{f\left(P_{1} \oplus P_{2}\right)}}
$$

where $f(\cdot)$ and $\mu_{0}(\cdot)$ are the frequency and the mean under null hypothesis of independence of two phrases (El-Kishky et al. 2014). $\oplus$ is the concatenation operator. The equation computes the number of standard deviations away from the expected number of occurrences, and this score can be considered a generalization of the $t$ statistic for identifying dependent bigrams. To extend the identified phrases to its bilingual, we first derive the word alignment information for the bilingual data using the model proposed by Dyer et al. (2013). The word alignments act as vital infor- 
mation and are used to project the phrase boundaries from the source sentences to the target side of the bilingual data (Zeng et al., 2014). For those of unaligned words or phrases, we simply ignore it from the induction process. The described bilingual phrases extraction model is a kind of unsupervised approach, where all the statistics are automatically derived from a given parallel corpus aligned at sentence level.

The bilingual multi-word expressions obtained are fed into an online translation engine for further automatic evaluation. Each monolingual part of a pair of bilingual terms is input to a translation engine to obtain a translation, which is compared with the other part of the bilingual pair in terms of Levenshtein distance (LD). The following results are obtained: $\mathrm{LD}=0: 11.9 \%, \mathrm{LD}=1: 25.5 \% ; \mathrm{LD}=2: 29.3 \%$; $\mathrm{LD}=3: 11.9 \%$; $\mathrm{LD}=4: 11.0 \% ; \mathrm{LD} \geq 5: 10.3 \%$.

We noted that from the figures, only $11.9 \%$ are identical with online translation result. The remaining near $90 \%$ are not identical but are still potentially valid entries, and are more valuable because they reflect the actual language in this particular domain which are not readily manifested through any publicly available resources. Further empirical studies show that lower edit-distance entries require less manual modifications. When the edit distance is equal to 1 or 2 , about $65 \%$ are valid entries without modifications. $5 \%$ can be useful after manual modifications. For distance 3 or 4 , about $55 \%$ are valid entries, while about $10 \%$ can become useful after modifications.

Our efforts have produced over 6 million MWE candidate entries. Further mostly straightforward manual efforts have yielded 1 million good bilingual MWEs thus far with another 1.5 million expected on completion of the project. The human efforts mostly entail the pruning of redundant constituents and in some cases the recovery of missing constituents as given in the examples in section 3 . The final harvest rate should be about $40 \%$ or approximately 2.5 million bilingual MWEs.

\section{Concluding Remarks}

We have shown that highly selective bilingual multiword expressions in the field of Chinese-English patents can be rigorously mined from a much larger and carefully cultivated database of comparable patent consisting of more than $300+\mathrm{k}$ ChineseEnglish patents involving more than 10.5 trillion Chinese characters. They can be highly useful for efforts in computer-assisted translation and cross-lingual information retrieval, for example. Several areas continue to pose challenges in the expeditious cultivation of these bilingual terms: (1) inherent noise in data because of OCR errors; (2) constituent redundancy, including cases of numbers, letters, modification and punctuation;(3) constituent omission, including cases of missing headword, preheaded/post-headed modification, and incomplete sentences.

Resolving these challenges will enhance the semi-automatic cultivation of linguistically sound multiword expressions. Computational methods seem to plateau at a certain level regarding many of these linguistic issues and human efforts are still needed at present stage, and this remains an open research area. 


\section{References}

1. Dyer, Chris, Victor Chahuneau, and Noah A. Smith.: A simple, fast, and effective reparameterization of IBM Model 2. Proceedings of NAACL-HLT, pp. 644-648. (2013).

2. El-Kishky, Ahmed, Yanglei Song, Chi Wang, Clare R. Voss, and Jiawei Han.: Scalable Topical Phrase Mining from Text Corpora. PVLDB 8(3), 305-316(2014).

3. Goto, Isao, Ka Po Chow, Bin Lu, Eiichiro Sumita, and Benjamin K. Tsou.: Overview of the patent machine translation task at the NTCIR-10 workshop. Proceedings of NTCIR-10 Workshop Meeting. (2013).

4. Koehn, Philipp.:Statistical machine translation. Cambridge University Press, the United Kingdom (2010).

5. Liu, Jialu, Jingbo Shang, Chi Wang, Xiang Ren, and Jiawei Han.: Mining Quality Phrases from Massive Text Corpora. Proceedings of the 2015 ACM SIGMOD International Conference on Management of Data. pp. 1729-1744. Melbourne, Victoria, Australia (2015).

6. Lu, Bin and Benjamin K. Tsou.: Towards Bilingual Term Extraction in Comparable Patents. In Proceedings of the 23rd Pacific Asia Conference on Language, Information and Computation (PACLIC'23). (2009).

7. Lu, Bin, Benjamin K. Tsou, Jingbo Zhu, Tao Jiang, and Olivia Y. Kwong.: The Construction of an English-Chinese Patent Parallel Corpus. MT Summit XII 3rd Workshop on Patent Translation. (2009).

8. Lu, Bin, Benjamin K. Tsou, Tao Jiang, Oi Yee Kwong and Jingbo Zhu.: Mining Largescale Parallel Corpora from Multilingual Patents: An English-Chinese example and its application to SMT. In Proceedings of the 1st CIPS-SIGHAN Joint Conference on Chinese Language Processing (CLP-2010). Beijing, China (2010a).

9. Lu, Bin, Tao Jiang, Kapo Chow and Benjamin K. Tsou.: Building a Large English-Chinese Parallel Corpus from Comparable Patents and its Experimental Application to SMT. In Proceedings of the Workshop on Building and Using Comparable Corpora. (2010b).

10. Lu, Bin, Benjamin K. Tsou, Tao Jiang, Jingbo Zhu and Olivia Kwong.: Mining Parallel Knowledge from Comparable Patents. In: Ontology Learning and Knowledge Discovery Using the Web: Challenges and Recent Advances. IGI Global. (2011).

11. Lu Bin, Chow K.P., Tsou B.K.: Comparable Multilingual Patents as Large-Scale Parallel Corpora. In: Sharoff S., Rapp R., Zweigenbaum P., Fung P. (eds) Building and Using Comparable Corpora. Springer, Heidelberg (2013).

12. Lu Bin, Benjamin K. Tsou and Ka Po Chow.: Cultivating Large-scale Parallel Corpora from Comparable Patents: From Bilingual to Trilingual, and Beyond. Linguistic Corpus and Corpus Linguistics in the Chinese Context (Journal of Chinese Linguistics Monograph Series No.25)(Tsou, Benjamin, and Kwong, Olivia. (Eds).), 334-355 (2015).

13. Ma, Xiaoyi.: Champollion: A Robust Parallel Text Sentence Aligner. In Proceedings of the 5th International Conference on Language Resources and Evaluation (LREC). Genova, Italy (2006).

14. Tian, Liang, Fai Wong, and Sam Chao.: Phrase Oriented Word Alignment Method. In Wang, Hai Feng (Ed.), Proceedings of the 7th China Workshop on Machine Translation pp. 237-250. Xiamen, China (2011).

15. Tian, Liang, Derek F. Wong, Lidia S. Chao, and Francisco Oliveira.: A Relationship: Word Alignment, Phrase Table, and Translation Quality. The Scientific World Journal113 (2014).

16. Tsou, Benjamin K.: Challenges And Advances IN Natural Language Processing of Chinese Patents-From Machine Translation To Cognitive Filtering. Invited paper, East meets West Forum, 2017 European Patent Office, Vienna (2017). 
17. Utiyama, Masao and Hitoshi Isahara.: Reliable measures for aligning japanese-english news articles and sentences. In Proceedings of the 41st Annual Meeting of the Association for Computational Linguistics, pp. 72- 79. Sapporo, Japan (2003).

18. Zeng, Xiaodong, Lidia S. Chao, Derek F. Wong, Isabel Trancoso, and Liang Tian.: Toward Better Chinese Word Segmentation for SMT via Bilingual Constraints. Proceedings of the 52nd Annual Meeting of the Association for Computational Linguistics (Volume 1: Long Papers), pp. 1360-1369. Baltimore, Maryland (2014). 


\section{Appendix:}

Filtering of Bilingual Chinese-English Multi-word Expressions from Large Scale Parallel Corpora of Comparable Patents

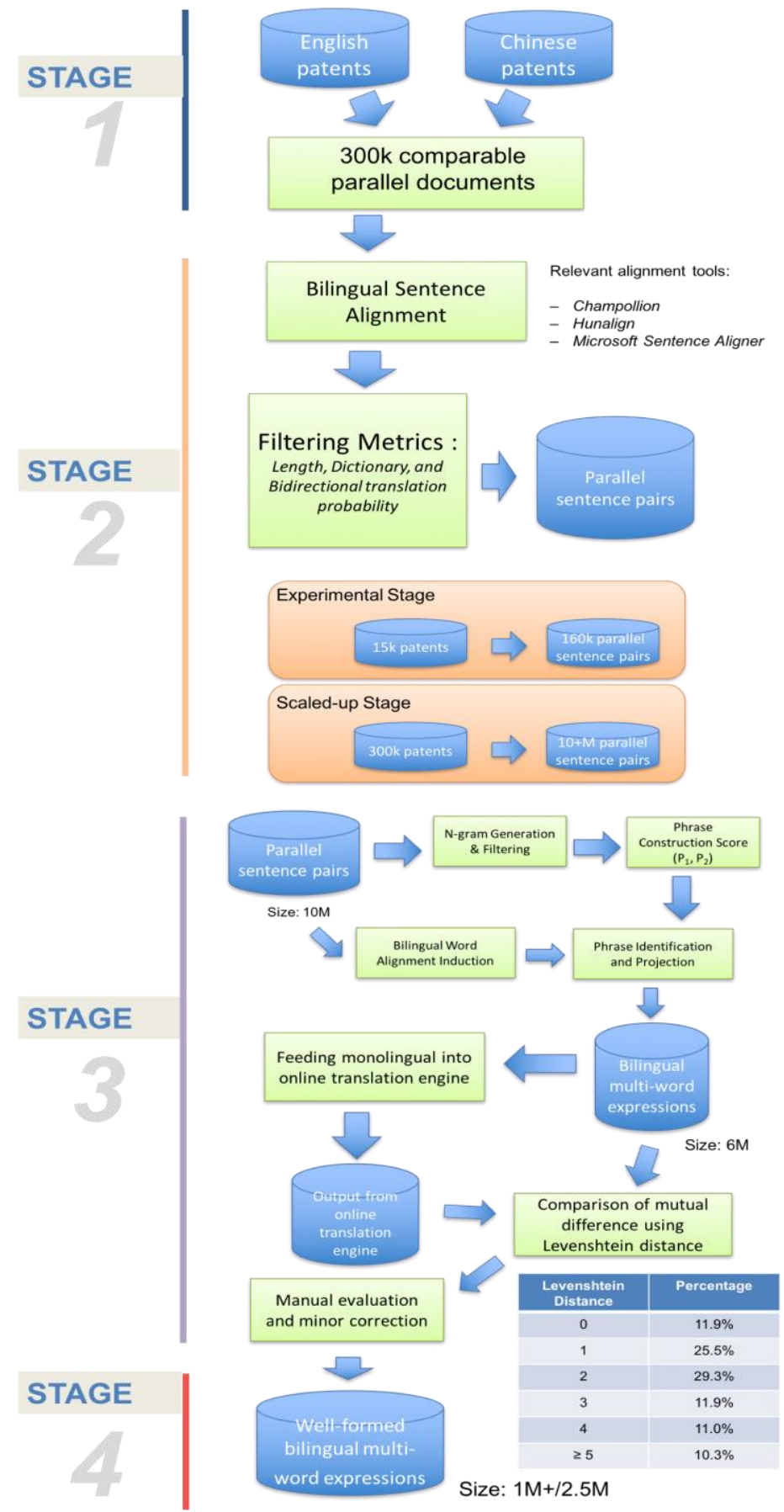

